\title{
Kantorovich-Schurer bivariate operators
}

Dan Bărbosu 


\title{
KANTOROVICH-SCHURER BIVARIATE OPERATORS
}

\author{
DAN BĂRBOSU
}

[Received: April 30, 2003]

$$
\begin{aligned}
& \text { ABstract. Let } p, q \text { be two non-negative given integers. The sequence }\left(\tilde{K}_{m, n, p, q}\right)_{m, n \in \mathbb{N}}, \\
& \tilde{K}_{m, n, p, q}: L_{1}([0,1] \times[0,1]) \rightarrow C([0,1] \times[0,1]), \\
& \qquad \begin{aligned}
\left(\tilde{K}_{m, n, p, q} f\right)(x, y) & =(m+p+1)(n+p+1) \\
& \times \sum_{k=0}^{m+p} \sum_{j=0}^{n+q} \tilde{p}_{m, k}(x) \tilde{p}_{n j}(y) \int_{\frac{k}{m+p+1}}^{\frac{k+1}{m+p+1}} \int_{\frac{j}{n+q+1}}^{\frac{j+1}{n+q+1}} f(s, t) d s d t
\end{aligned}
\end{aligned}
$$

of bivariate Kantorovich-Schurer operators is constructed and some approximation properties of the sequence $\left\{\tilde{K}_{m, n, p, q} f\right\}_{m, n \in \mathbb{N}}$ are studied.

Mathematics Subject Classification: 41A36, 41A63

Keywords: Linear positive operator, Bernstein operator, Kantorovich operator, Schurer operator, bivariate operator, Korovkin theorem, first order modulus of smoothness, Shisha-Mond theorem

\section{Preliminaries}

STARTING with the well-known Bernstein operator $B_{m}$, L. V. Kantorovich [6] introduced and studied the operator $K_{m}: L_{1}([0,1]) \rightarrow C([0,1])$ defined for any $m \in \mathbb{N}$ and any $f \in L_{1}([0,1])$ by

$$
\left(K_{m} f\right)(x)=(m+1) \sum_{k=0}^{m} p_{m, k}(x) \int_{\frac{k}{m+1}}^{\frac{k+1}{m+1}} f(t) d t,
$$

where $p_{m, k}(x)=\left(\begin{array}{c}m \\ k\end{array}\right) x^{k}(1-x)^{m-k}$ are the fundamental Bernstein polynomials. Operators (1.1) are known in mathematical literature as the Kantorovich operators.

In 1962, F. Schurer [9] considering a given non-negative integer $p$ constructed and studied a generalization of classical Bernstein operator. This generalization is the operator $\tilde{B}_{m, p}: C([0,1+p]) \rightarrow C([0,1])$ defined for any $m \in \mathbb{N}$ and any $f \in$ $C([0,1+p])$ by

$$
\left(\tilde{B}_{m, p} f\right)(x)=\sum_{k=0}^{m+p} \tilde{p}_{m, k}(x) f\left(\frac{k}{m}\right),
$$


where $\tilde{p}_{m, k}(x)=\left(\begin{array}{c}m+p \\ k\end{array}\right) x^{k}(1-x)^{m+p-k}$ are the fundamental Schurer polynomials.

Starting with the operator (1.2), in [4], we constructed the Kantorovich-Schurer operator $\tilde{K}_{m, p}: L_{1}([0,1]) \rightarrow C([0,1])$, defined for any $m \in \mathbb{N}$ and any $f \in L_{1}([0,1])$ by

$$
\left(\tilde{K}_{m, p} f\right)(x)=(m+p+1) \sum_{k=0}^{m+p} \tilde{p}_{m, k}(x) \int_{\frac{k}{m+p+1}}^{\frac{k+1}{m+p+1}} f(t) d t
$$

There, we established a convergence theorem for the sequence $\left\{\tilde{K}_{m, p} f\right\}$ and we gave quantitative estimations of the approximation order in terms of first order modulus of smoothness.

Considering two given non-negative integers $p$ and $q$, in [2], we constructed the bivariate Schurer operator $\tilde{B}_{m, n, p, q}: C([0,1+p] \times[0,1+q]) \rightarrow C([0,1] \times[0,1])$

$$
\left(\tilde{B}_{m, n, p, q} f\right)(x, y)=\sum_{k=0}^{m+p} \sum_{j=0}^{n+q} \tilde{p}_{m, k}(x) \tilde{p}_{n, j}(y) f\left(\frac{k}{m}, \frac{j}{n}\right) .
$$

The purposes of the present paper are the following:

(i) To construct the bivariate Kantorovich-Schurer operator

$$
\tilde{K}_{m, n, p, q}: L_{1}([0,1] \times[0,1]) \rightarrow C([0,1] \times[0,1]) ;
$$

(ii) To establish a convergence theorem for the sequence $\left\{\tilde{K}_{m, n, p, q} f\right\}_{m, n \in \mathbb{N}}$;

(iii) To give quantitative estimations of the approximation order in terms of first order modulus of smoothness for bivariate functions.

\section{The CONSTRUCtion of Bivariate Kantorovich-Schurer OPERATORS}

Let $p \geq 0, q \geq 0$ be two given integers and let $\tilde{K}_{m, p}: L_{1}([0,1]) \rightarrow C([0,1]), \tilde{K}_{n, q}$ : $L_{1}([0,1]) \rightarrow C([0,1])$ defined for any $m, n \in \mathbb{N}$ and any $g \in L_{1}([0,1]), h \in L_{1}([0,1])$, respectively, by:

$$
\left(\tilde{K}_{m, p} g\right)(x)=(m+p+1) \sum_{k=0}^{m+p} \tilde{p}_{m, k}(x) \int_{\frac{k}{m+p+1}}^{\frac{k+1}{m+p+1}} g(s) d s
$$

and

$$
\left(\tilde{K}_{n, q} h\right)=(n+q+1) \sum_{j=0}^{n+q} \tilde{p}_{n, j}(y) \int_{\frac{j}{n+q+1}}^{\frac{j+1}{n+q+1}} h(t) d t,
$$

where $\tilde{p}_{m, k}(x), \tilde{p}_{n, j}(y)$ are the fundamental Schurer polynomials.

The parametric extensions (for this term, see [3] or [5]) of (2.1) and (2.2) are the operators $\tilde{K}_{m, p}^{x}, \tilde{K}_{n, q}^{y}: L_{1}([0,1] \times[0,1]) \rightarrow C([0,1] \times[0,1])$, defined for any $m, n \in \mathbb{N}$ 
and any $f \in L_{1}([0,1] \times[0,1])$ as follows:

$$
\begin{gathered}
\left(\tilde{K}_{m, p}^{x} f\right)(x, y)=(m+p+1) \sum_{k=0}^{m+p} \tilde{p}_{m, k}(x) \int_{\frac{k}{m+p+1}}^{\frac{k+1}{m+p+1}} f(s, y) d s, \\
\left(\tilde{K}_{n, j}^{y} f\right)(x, y)=(n+q+1) \sum_{j=0}^{n+q} \tilde{p}_{n, j}(y) \int_{\frac{j}{n+q+1}}^{\frac{j+1}{n+q+1}} f(x, t) d t .
\end{gathered}
$$

Lemma 2.1. The parametric extensions of the univariate Kantorovich-Schurer operator, defined by (2.3) and (2.4) are linear and positive operators.

Proof. The assertion follows from the definitions of $\tilde{K}_{m, p}^{x}$ and $\tilde{K}_{n, q}^{y}$.

Lemma 2.2. The parametric extensions of the Kantorovich-Schurer operator commute on $L_{1}([0,1] \times[0,1])$. Their product is the bivariate Kantorovich-Schurer operator $\tilde{K}_{m, n, p, q}: L_{1}([0,1] \times[0,1]) \rightarrow C([0,1] \times[0,1])$ defined for any $m, n \in \mathbb{N}$ and any $f \in L_{1}([0,1] \times[0,1])$ by the relation

$$
\begin{aligned}
\left(\tilde{K}_{m, n, p, q} f\right)(x, y)=(m & +p+1)(n+q+1) \\
& \times \sum_{k=0}^{m+p} \sum_{j=0}^{n+q} \tilde{p}_{m, k}(x) \tilde{p}_{n, j}(y) \int_{\frac{k}{m+p+1}}^{\frac{k+p}{m+1}} \int_{\frac{j}{n+q+1}}^{\frac{j+1}{n+q+1}} f(s, t) d s d t .
\end{aligned}
$$

Proof. We arrive to the desired result by direct computation, taking into account definitions (2.3), (2.4) and Lemma 2.1.

Lemma 2.3. The bivariate Kantorovich-Schurer operator (2.5) is linear and positive.

Proof. The product of linear and positive linear operators is a linear and positive operator. We apply next Lemma 2.1 .

\section{ConvergenCe PROPERTIES OF THE SEQUence $\left\{\tilde{K}_{m, n, p, q} f\right\}_{m, n \in \mathbb{N}}$}

In what follows, $e_{i j}(x, y)=x^{i} y^{j}(i, j \in \mathbb{N}, 0 \leq i+j \leq 2)$ denotes the test functions. We need the following auxiliary result (the first Korovkin Theorem for approximation of bivariate continuous functions [12]).

Theorem 3.1. Let $a, b, c, d$ be real numbers satisfying the inequalities $a<b$, $c<d$ and let $\left(L_{m, n}\right)_{m, n \in \mathbb{N}}$ be a sequence of linear and positive operators $L_{m, n}$ : $C([a, b] \times[c, d]) \rightarrow C([a, b] \times[c, d])$ having the properties

$$
\begin{gathered}
\left(L_{m, n} e_{00}\right)(x, y)=1+u_{m, n}(x, y), \\
\left(L_{m, n}\left(e_{10}-x\right)^{2}\right)(x, y)=v_{m, n}(x, y), \\
\left(L_{m, n}\left(e_{01}-y\right)^{2}\right)(x, y)=w_{m, n}(x, y) .
\end{gathered}
$$


If

$$
\lim _{m, n \rightarrow \infty} u_{m, n}(x, y)=\lim _{m, n \rightarrow \infty} v_{m, n}(x, y)=\lim _{m, n \rightarrow \infty} w_{m, n}(x, y)=0
$$

uniformly on $[a, b] \times[c, d]$, then the sequence $\left\{L_{m, n} f\right\}_{m, n \in \mathbb{N}}$ converges to $f$ uniformly on $[a, b] \times[c, d]$ as $m, n \rightarrow \infty$.

In our earlier paper [4], we proved

Lemma 3.1 (Lemma 3 in [4]). For any $x \in[0,1+p]$, the operator $\tilde{K}_{m, p}$ satisfies the relations

$$
\begin{aligned}
& \left(\tilde{K}_{m, p} e_{0}\right)(x)=1 \\
& \left(\tilde{K}_{m, m} e_{1}\right)(x)=\frac{m+p}{m+p+1} x+\frac{1}{2(m+p+1)}, \\
& \left(\tilde{K}_{m, p} e_{2}\right)(x)=\frac{m+p}{(m+p+1)^{2}}\left\{(m+p) x^{2}+x(2-x)\right\}+\frac{1}{3(m+p+1)^{2}} .
\end{aligned}
$$

Lemma 3.2. The parametric extension $\tilde{K}_{m, p}^{x}$ satisfies the identities (3.4), (3.5), and (3.6).

Proof. We make use of the definition (2.3) of $\tilde{K}_{m, p}^{x}$ and of Lemma 3.1.

Remark 3.1. The parametric extension $\tilde{K}_{n, q}^{y}$ satisfies identities similar to the identities (3.4), (3.5), and (3.6).

Lemma 3.3. The bivariate Kantorovich-Schurer operator $\tilde{K}_{m, n, p, q}$ defined by (2.5) satisfies the equalities

$$
\begin{aligned}
\left(\tilde{K}_{m, n, p, q} e_{00}\right)(x, y) & =1 \\
\left(\tilde{K}_{m m, n, p, q} e_{10}\right)(x, y) & =\frac{m+p}{m+p+1} x+\frac{1}{2(m+p+1)}, \\
\left(\tilde{K}_{m, n, p, q} e_{01}\right)(x, y) & =\frac{n+q}{n+q+1} y+\frac{1}{2(n+q+1)}, \\
\left(\tilde{K}_{m, n, p, q} e_{20}\right)(x, y) & =\frac{m+p}{(m+p+1)^{2}}\left\{(m+p) x^{2}+x(2-x)\right\} \\
& +\frac{1}{3(m+p+1)^{2}}, \\
\left(\tilde{K}_{m, n, p, q} e_{02}\right)(x, y) & =\frac{n+q}{(n+q+1)^{2}}\left\{(n+q) y^{2}+y(2-y)\right\} \\
& +\frac{1}{3(n+q+1)^{2}} .
\end{aligned}
$$

Proof. Taking into account definition (2.5) and Lemma 3.2, we arrive at the desired equalities. 
Lemma 3.4. The bivariate Kantorovich-Schurer operator (2.5) satisfies the relations

$$
\begin{aligned}
& \left(\tilde{K}_{m, n, p, q}\left(e_{10}-x\right)^{2}\right)(x, y)=\frac{m+p-1}{(m+p+1)^{2}} x(1-x)+\frac{1}{3(m+p+1)^{2}}, \\
& \left(\tilde{K}_{m, n, p, q}\left(e_{01}-y\right)^{2}\right)(x, y)=\frac{n+q-1}{(n+q+1)^{2}} y(1-y)+\frac{1}{3(n+q+1)^{2}} .
\end{aligned}
$$

Proof. Since $\tilde{K}_{m, n, p, q}$ is linear, we have

$$
\begin{aligned}
\left(\tilde{K}_{m, n, p, q}\left(e_{10}-x\right)^{2}\right)(x, y) & =\left(\tilde{K}_{m, n, p, q} e_{20}\right)(x, y) \\
& -2 x\left(\tilde{K}_{m, n, p, q} e_{10}\right)(x, y)+x^{2}\left(\tilde{K}_{m, n, p, q} e_{10}\right)(x, y) .
\end{aligned}
$$

Next, applying Lemma 3.3, we get relation (3.12). Equality (3.13) is proved in a similar way.

Theorem 3.2. The sequence $\left\{\tilde{K}_{m, n, p, q} f\right\}_{m, n \in \mathbb{N}}$ converges to $f$ uniformly on $[0,1] \times$ $[0,1]$ for any $f \in L_{1}([0,1] \times[0,1])$.

Proof. We apply Theorem 3.1 with

$$
\begin{aligned}
& u_{m, n}(x, y)=0, \\
& v_{m, n}(x, y)=\frac{m+p-1}{(m+p+1)^{2}} x(1-x)+\frac{1}{3(m+p+1)^{2}}, \\
& w_{m, n}(x, y)=\frac{n+q-1}{(n+q+1)^{2}} y(1-y)+\frac{1}{3(n+q+1)^{2}} .
\end{aligned}
$$

\section{Estimation OF THE RATE OF CONVERGENCE}

Here, we focus on estimating the rate of convergence for $\left\{\tilde{K}_{m, n, p, q} f\right\}$ in terms of the first order modulus of smoothness for bivariate functions. We first recall the following.

Definition 4.1. Let $a, b, c, d \in \mathbb{R}$ be given so that $a<b, c<d$ and let $f:[a, b] \times$ $[c, d] \rightarrow \mathbb{R}$ be a bounded function. The function $\omega_{f}:[0, b-a] \times[0, d-c] \rightarrow \mathbb{R}$, defined for any $\left(\delta_{1}, \delta_{2}\right) \in[0, b-a] \times[0, d-c]$

$$
\begin{aligned}
\omega_{f}\left(\delta_{1}, \delta_{2}\right)=\sup \left\{\left|f(x, y)-f\left(x^{\prime}, y^{\prime}\right)\right|:(x, y),\right. \\
\left.\quad\left(x^{\prime}, y^{\prime}\right) \in[0, b-a] \times[0, d-c],\left|x-x^{\prime}\right| \leq \delta_{1},\left|y-y^{\prime}\right| \leq \delta_{2}\right\}
\end{aligned}
$$

is called first order modulus of smoothness of function $f$.

The first order modulus of smoothness for bivariate functions has properties similar to the properties of the first order modulus of smoothness for univariate functions. Some of them are contained in 
Lemma 4.1. The first order modulus of smoothness for bivariate functions (4.1) has the following properties:

(i) $\omega_{f}\left(\delta_{1}, \delta_{2}\right) \leq \omega_{f}\left(\delta_{1}^{\prime}, \delta_{2}^{\prime}\right)$ for all $\left(\delta_{1}, \delta_{2}\right)$ and $\left(\delta_{1}^{\prime}, \delta_{2}^{\prime}\right)$ from $[0, b-a] \times[0, d-c]$ such that $\delta_{1}<\delta_{1}^{\prime}$ and $\delta_{2}<\delta_{2}^{\prime}$;

(ii) $\omega_{f}\left(\lambda_{1} \delta_{1}, \lambda_{2} \delta_{2}\right) \leq\left(1+\lambda_{1}\right)\left(1+\lambda_{2}\right) \omega_{f}\left(\delta_{1}, \delta_{2}\right)$ for all $\left(\delta_{1}, \delta_{2}\right) \in[0, b-a] \times[0, d-c]$ and $\lambda_{1}, \lambda_{2} \in \mathbb{R}_{+}^{*}$.

The following version of the Shisha-Mond theorem [12] for estimating the rate of convergence is known.

Theorem 4.1. Let $\left(L_{m, n}\right)_{m, n \in \mathbb{N}}$ be a sequence of bivariate linear positive operators mapping the space $C([a, b] \times[c, d])$ into itself and reproducing the constant functions. Then, for any $f \in C([a, b] \times[c, d])$ and any $\left(\delta_{1}, \delta_{2}\right) \in[0, b-a] \times[0, d-c]$, the estimate

$$
\begin{aligned}
\left|L_{m, n} f(x, y)-f(x, y)\right| \leq & \left\{1+\delta_{1}^{-1} \sqrt{\left(L_{m, n}\left(e_{10}-x\right)^{2}\right)(x, y)}+\right. \\
& +\delta_{2}^{-1} \sqrt{\left(L_{m, n}\left(e_{01}-y\right)^{2}\right)(x, y)}+ \\
+ & \left.\delta_{1}^{-1}, \delta_{2}^{-1} \sqrt{\left(L_{m, n}\left(e_{10}-x\right)^{2}\right)(x, y) \cdot\left(L_{m, n}\left(e_{01}-y\right)^{2}\right)(x, y)}\right\} \cdot \omega_{f}\left(\delta_{1}, \delta_{2}\right)
\end{aligned}
$$

is true.

Now, we are ready to prove the main result of this section.

Theorem 4.2. For any $f \in C([0,1] \times[0,1])$ and any $(x, y) \in[0,1] \times[0,1]$, the operator $\tilde{K}_{m, n, p, q}$ satisfies the relation

$$
\begin{aligned}
& \left|\tilde{K}_{m, n, p, q} f(x, y)-f(x, y)\right| \leq \\
& \leq 4 \omega_{f}\left(\frac{\sqrt{3(m+p-1) x(1-x)+1}}{\sqrt{3}(m+p+1)}, \frac{\sqrt{3(n+q-1) y(1-y)+1}}{\sqrt{3}(n+q+1)}\right) \\
& \quad \leq 4 \omega_{f}\left(\frac{\sqrt{3 m+3 p+1}}{2 \sqrt{3}(m+p+1)}, \frac{\sqrt{3 n+3 q+1}}{2 \sqrt{3}(n+q+1)}\right) .
\end{aligned}
$$

Proof. Applying Theorem 4.1 and Lemma 3.4, we get

$$
\begin{aligned}
& \left|\tilde{K}_{m, n, p, q} f(x, y)-f(x, y)\right| \leq \\
& \quad \leq\left\{1+\frac{\delta_{1}^{-1}}{\sqrt{3}(m+p+1)} \sqrt{3(m+p+1) x(1-x)+1}+\right. \\
& +\frac{\delta_{2}^{-1}}{\sqrt{3}(n+q+1)} \sqrt{3(n+q+1) y(1-y)+1}+\frac{\delta_{1}^{-1} \delta_{2}^{-1}}{3(m+p+1)(n+q+1)} \times \\
& \quad \times \sqrt{(3(m+p+1) x(1-x)+1)(3(n+q+1) y(1-y)+1)}\} \omega_{f}\left(\delta_{1}, \delta_{2}\right)
\end{aligned}
$$


for any $\left(\delta_{1}, \delta_{2}\right) \in[0,1+p] \times[0,1+q]$. Choosing in (4.4)

$$
\delta_{1} \frac{\sqrt{3(m+p+1) x(1-x)+1}}{\sqrt{3}(m+p+1)}, \quad \delta_{2}=\frac{\sqrt{3(n+q+1) y(1-y)+1}}{\sqrt{3}(n+q+1)},
$$

we get the first inequality (4.3). Taking into account that $x(1-x) \leq 1 / 4, y(1-y) \leq 1 / 4$ for any $(x, y) \in[0,1] \times[0,1]$, we obtain the second inequality (4.3).

\section{The bivariate Kantorovich OPERators}

The Kantorovich bivariate operator $K_{m, n}: L_{1}([0,1] \times[0,1]) \rightarrow C([0,1] \times[0,1])$, defined for any $f \in L_{1}([0,1] \times[0,1])$ and any $m, n \in \mathbb{N}$ by the formula

$$
\left(K_{m, n} f\right)(x, y)=(m+1)(n+1) \sum_{k=0}^{m} \sum_{j=0}^{n} p_{m k}(x) p_{n j}(y) \int_{\frac{k}{m+1}}^{\frac{k+1}{m+1}} \int_{\frac{j}{n+1}}^{\frac{j+1}{n+1}} f(s, t) d s d t
$$

is the Kantorovich-Schurer bivariate operator $\tilde{K}_{m, n, 0,0}$. All the proprieties of $K_{m, n}$ can be obtained from the properties of $\tilde{K}_{m, n, p, q}$ for $p=q=0$. We formulate them as corollaries of the corresponding properties of $\tilde{K}_{m, n, p, q}$.

Corollary 5.1. The sequence $\left\{K_{m, n} f\right\}_{m, n \in \mathbb{N}}$ converges to $f$, uniformly on $[0,1] \times$ $[0,1]$, for any $f \in L_{1}([0,1] \times[0,1])$.

Proof. The assertion follows from Theorem 3.2 for $p=q=0$.

Corollary 5.2. For any $f \in C([0,1] \times[0,1])$ and any $(x, y) \in[0,1] \times[0,1]$, the Kantorovich bivariate operator (5.1) satisfies the relation

$$
\begin{aligned}
& \left|\left(K_{m, n} f\right)(x, y)-f(x, y)\right| \leq \\
& \quad \leq 4 \omega_{f}\left(\frac{\sqrt{3(m-1) x(1-x)+1}}{\sqrt{3}(m+1)}, \frac{\sqrt{3(n-1) y(1-y)+1}}{\sqrt{3}(n+1)}\right) \leq \\
& \quad \leq 4 \omega_{f}\left(\frac{\sqrt{3 m+1}}{2 \sqrt{3}(m+1)}, \frac{\sqrt{3 n+1}}{2 \sqrt{3}(n+1)}\right) .
\end{aligned}
$$

Proof. Application of Theorem 4.2 with $p=q=0$.

\section{REFERENCES}

[1] Agratinı, O.: Aproximare prin operatori liniari, Presa Universitară Clujeană, Cluj-Napoca 2000 (in Romanian).

[2] B̆̆rвosu, D.: Bivariate Bernstein-Schurer operators (to appear in Rev. Anal. Numer. Theor. Approx.)

[3] B̆̆RBosu, D.: Aproximarea functiilor de mai multe variabile prin sume booleene de operatori liniari de tip interpolator, Ed. Risoprint, Cluj-Napoca, 2002 (in Romanian).

[4] B̆̆Rbosu, D.: Kantorovich-Schurer operators (to appear in Novi-Sad Journal of Mathematics)

[5] Delvos, F. and Schempr, W.: Boolean methods in Interpolation and Approximation, Longman Scientific Technical, Harlow, 1989. 
[6] Kantorovich, L. V.: Sur certains développements suivant les polynômes de la forme de S. Bernstein, I, II, C. R. Acad. URSS (1930), 563-568, 559-600.

[7] LoRentz, G. G.: Bernstein polynomials, Mathematical Expositions, No. 8, University of Toronto Press, Toronto, 1953.

[8] Shisha, O. AND Mond, B.: The degree of convergence of linear positive operators, Proc. Nat. Acad. Sci., USA (1960), 1196-2000.

[9] Schurer, F.: Linear positive operators in approximation theory, Math. Inst. Tech. Univ. Delft. Report, 1962.

[10] Stancu, D. D.: Curs şi culegere de probleme de analiză numerică, lito. Univ. Babeş-Bolyai, ClujNapoca, 1977 (in Romanian).

[11] Stancu, D.D., Coman, Gh., Agratini, O. and Trîmbiţaş, R.: Analiză numerică şi teoria aproximării, vol. I, Presa Universitară Clujeană, Cluj-Napoca, 2001 (in Romanian).

[12] Stancu, F.: Aproximarea funcţiilor de două şi mai multe variabile cu ajutorul operatorilor liniari şi pozitivi, PhD Thesis, Univ. Babeş-Bolyai, Cluj-Napoca, 1984 (in Romanian).

[13] Тотік, V.: Approximation in $L^{1}$ by Kantorovich polynomials, Acta Sci. Math, 46 (1983), 211-222.

[14] Тотік, V.: Problems and solutions concerning Kantorovich operators, J. Approx. Theory, 37 (1983), 51-58.

\section{Author's Address}

\section{Dan Bărbosu:}

North University of Baia Mare, Faculty of Sciences, Department of Mathematics and Computer Science, Victoriei 76, 430122 Baia Mare, Romania

E-mail address: dbarbosu@ubm.ro; danbarbosu@yahoo.com 